\title{
The Charge Calibration of LHAASO-WCDA
}

\author{
Shicong Hu, ${ }^{a, 1, *}$ Yong Huang, ${ }^{a, 1}$ Chuandong Gao, ${ }^{b, 1}$ Zongkang Zeng, ${ }^{a, 1}$ Huicai Li, ${ }^{a, 1}$ \\ Bo Gao, ${ }^{a, 1}$ Cheng Liu, ${ }^{a, 1}$ Xiurong Li, ${ }^{a, 1}$ Min Zha $^{a, 1}$ and Zhiguo Yao ${ }^{a, 1}$ \\ ${ }^{a}$ Institute of High Energy Physics, Chinese Academy of Sciences, \\ 100049, Beijing, China \\ ${ }^{b}$ SHANDONG University, \\ 266237, Qingdao, China \\ E-mail: hushicong@ihep.ac.cn
}

The Water Cherenkov detector array (WCDA), one of major parts of LHAASO project, has been partly operated since April 2019. There are two types of PMT hung in center of each cell of WCDA. In order to achieve an optimal energy reconstruction, and cosmic ray background suppression, we develop an off-line method to calibrate number of photoelectrons of signals. By matching signals caught by different kinds of PMT, we bridge their linear measurement range to obtain equivalent NPE of signals up to more than $8 \times 10^{6}$ PEs for WCDA-1, and more than $1.5 \times 10^{5}$ PEs for WCDA-2. Besides, detector monitoring and various measurements show the PMT among cells are slightly different in quantum efficiency and collection efficiency, and the light attenuation and depth of water in the pool are also varying with time, especially in the very beginning of the operation. Above inhomogeneity and instablibility influence the detection efficiency of cells on secondary air shower particles. Based on previous research, the single particle peak in the charge spectrum of single cell mainly formed by muon signals are used to calibrate the detection efficiency difference as well as long term variation of all the detector cells. A possibly more flexible efficiency calibration method of Constant Rate Scaling is also under study. The analysis method and the calibration results of the first two pools are presented in this paper.

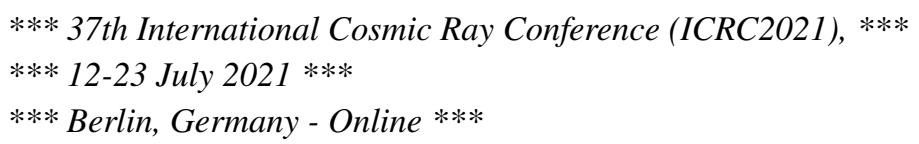

${ }^{1}$ For the LHAASO collaboration

*Presenter 


\section{Contents}

1 Introduction 2

2 Calibration of number of photo-electron 3

3 Efficiency calibration $\quad 5$

$\begin{array}{lll}4 & \text { Summary } & 7\end{array}$

\section{Introduction}

LHAASO-WCDA is a ground-based air-shower detector array based on water cherenkov technique conceived for investigating steady and transient VHE gamma-ray sources located at the northern sky from $0.1 \mathrm{TeV}$ to $30 \mathrm{TeV}$ [1]. As shown in Fig. 1, WCDA consists of three pools, two square pools, coevering each $22^{\prime} 500 \mathrm{~m}^{2}$, while a third rectangular one covers $33^{\prime} 000 \mathrm{~m}^{2}$. Each pool is divided into cells with area of $5 \mathrm{~m} \times 5 \mathrm{~m}$. There is an upward-looking 8-inch photomultiplier tube (PMT) and 1.5-inch PMT in each cell of WCDA-1, while a 20" PMT and 3" PMT are hung in each cell of WCDA-2 and WCDA-3. This combination of the two covers a wide dynamic range in terms of charge of signals, e.g. $1 \mathrm{PE}$ to $8 \times 10^{6} \mathrm{PEs}$ for combination of 8-inch and 1.5-inch, which allows a better measurement of secondary particle density distribution in the shower core without saturation.

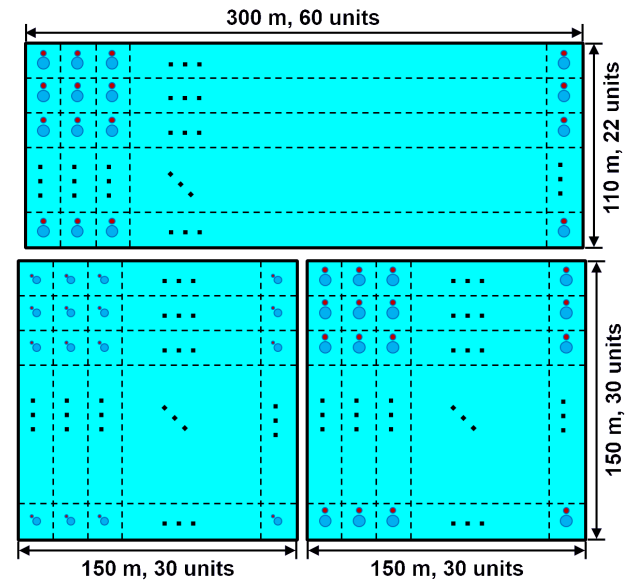

Figure 1: Schematic drawing of the WCDA layout.The left bottom one is WCDA-1, the right bottom is WCDA-2 and the top one is WCDA-3.

The monitoring of water level and attenuation length is important to understand change of detector running status. The measurement of attenuation length has been expounded in detail 
elsewhere [2]. As shown in Fig. 2, attenuation length of WCDA-1 gradually rose from beginning of 2019 December (330 $0^{\text {th }}$ days) and dropped rapidly from end of April 2020 (485 ${ }^{\text {th }}$ days). Because of leakage and aperiodic replenishment, change of water level over time is zigzag as shown in Fig. 3. The detection efficiency on secondary air shower particles is time dependent according to monitoring of water level and attenuation length. The performance of the detector depends critically on detection efficiency of the cells. And that is the motivation that lead us to develop a method named as Constant Rate Scaling (CRS) to calibrate detection efficiency of the cells.

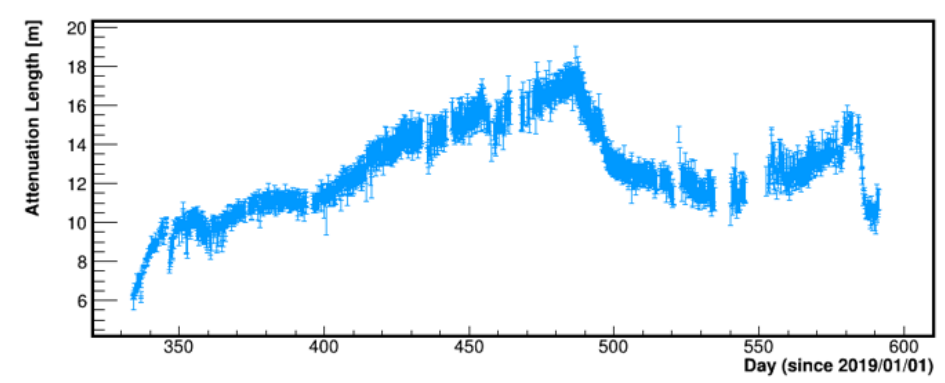

Figure 2: Change of attenuation length of WCDA-1 from 2019/12/01 to 2020/08/14.

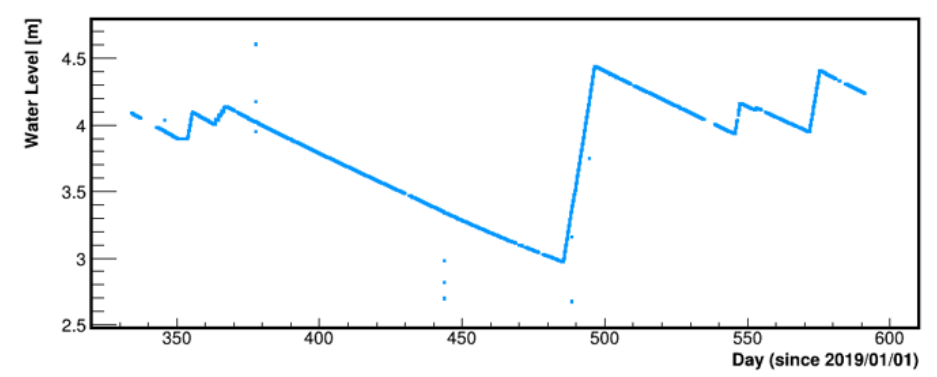

Figure 3: Variation of water level of WCDA-1 from 2019/12/01 to 2020/08/14.

\section{Calibration of number of photo-electron}

One of running modes of data acquisition system (DAQ) of LHAASO-WCDA is single-channel mode. In this mode, all hits of each cell are saved. The single channel charge spectrum of one sample 8-inch PMT is presented in Fig. 4, which is directly decoded from raw data. The peak around 25 ADC counts is mainly formed by single photon signals and its peak position is used to convert charge of signals from ADC counts to number of photoelectrons (NPEs). The 8-inch PMT is read out from both anode and $8^{t h}$ dynode to cover a range from $1 \mathrm{PE}$ to 4000 PEs. The range covered by anode channel is up to $130 \mathrm{PEs}$, with a non-linearity of less than 5\% [3]. Saturation exists when the anode signal exceeds 4096 ADC counts. The calibration of the dynode signals is performed by comparing them with the corresponding anode signals in the overlapping region. The ratio between the two signals denoted as $D / A$, can be extracted by fitting in the region of anode signals between 2400 and 3000 ADC counts. In Fig. 5(a), the dynode signal is plotted against the anode signal in terms of ADC counts for a typical 8-inch PMT. Distribution of $D / A$ ratio of 900 8-inch PMTs in WCDA-1 is presented in Fig. 5(b). A similar method is used to calibrate the $D / A$ ratio of three other kinds of PMT. By combining $6^{\text {th }}$ dynode with anode, dynamic range of 1.5 inch PMT is 20 
PEs - 200000 PEs, and non-linearity within this range is less than 5\% [4]. Calibration results of a typical 1.5-inch PMT and distribution of $D / A$ ratio of 900 1.5-inch PMT in WCDA-1 are shown in Fig. 5(c) and Fig. 5(d), respectively. Performance of four types of PMTs in LHAASO-WCDA are listed in Tab. 1, in which SPE and $D / A$ ratio are mean value of 900 PMTs in WCDA-1 or WCDA-2.

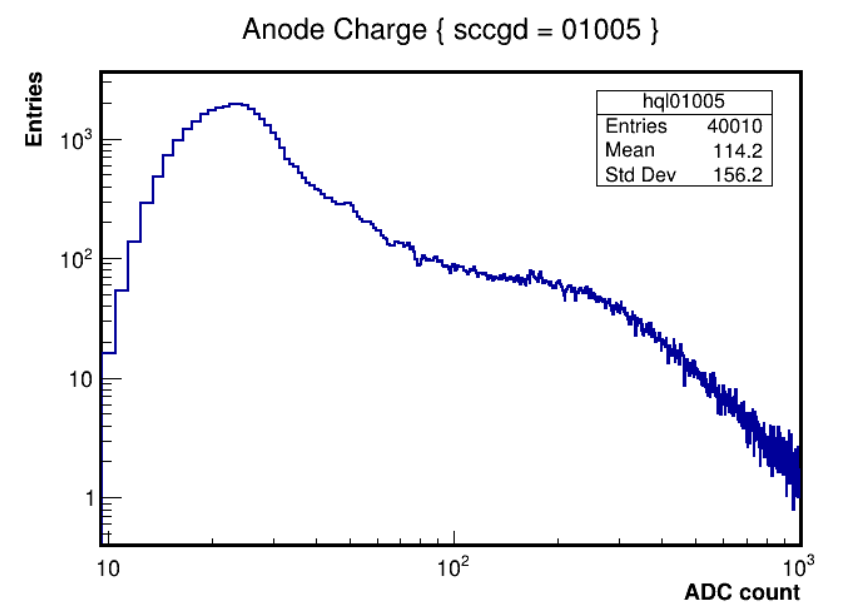

Figure 4: Single channel charge spectrum in cells of ADC conut.

\begin{tabular}{c|c|c|c} 
Type & SPE [ADC counts] & Dynamic range & $D / A$ ratio \\
\hline 8-inch & 26.7 & $1 \mathrm{PE}-4 \times 10^{3}$ PEs & 0.0225 \\
1.5-inch & - & $20 \mathrm{PEs}-2 \times 10^{5}$ PEs & 0.0186 \\
20-inch & 41.9 & $1 \mathrm{PE}-1.8 \times 10^{3}$ PEs & 0.0505 \\
3-inch & - & $1 \mathrm{PE}-3 \times 10^{3}$ PEs & 0.0286
\end{tabular}

Table 1: Performance of four types of PMTs in LHAASO-WCDA.

The calibration of 1.5-inch/3-inch PMTs has been performed by matching signals, which are produced by the same air shower event, with 8 -inch/20-inch PMTs in the same cell. The $A_{\text {plus }} / D$ ratio is given by linearly fitting the overlapping region of square root of dynode signals of 8-inch/20inch PMTs and that of anode signals of 1.5-inch/3-inch PMTs. As is shown in Fig. 6, the overlapping region is $2500 \mathrm{ADC}$ counts - $3025 \mathrm{ADC}$ counts for a combination of 8-inch PMT and 1.5-inch PMT and 2601 ADC counts - 3136 ADC counts for 20-inch PMT and 3-inch PMT. The mean value of $A_{\text {plus }} / D$ is 0.0240 and 0.0242 .

The final step is to connect signals of 20-inch PMTs in WCDA-2 to that of 8-inch PMTs in WCDA-1. Air shower events, of which core position falls in the middle of WCDA-1 and WCDA-2, are chose to calibrate the ratio of these two types of PMTs. The secondary particle density at the same distance from the core in the shower plane is considered to be statistically approximately equal. Therefore, in the case of no difference in water, the ratio of signal of a cell in WCDA-2 to that of a cell in WCDA-1 is the ratio of the corresponding 20-inch PMT to 8-inch PMT if the two cells are equal in distance from the core in the shower plane. Specifically, hits of one event are divided into nine groups according to the distance from the core. Each group of hits falls within the same loop with a width of 10 meters centered on the core. In the same loop, parts of hits are in 


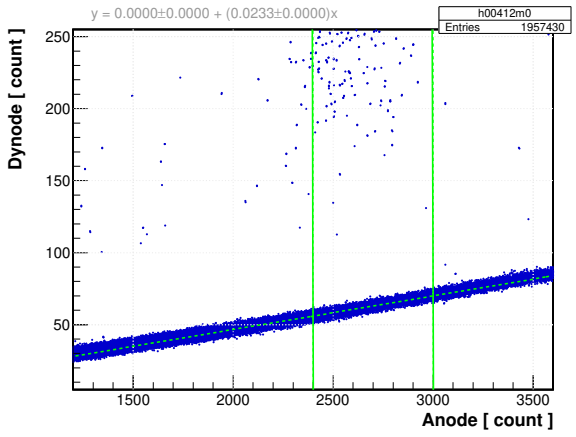

(a)

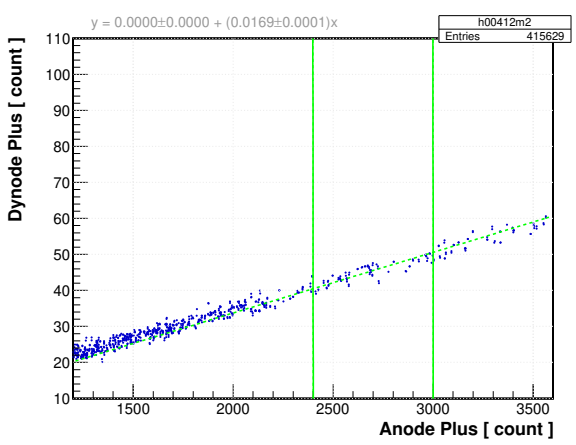

(c)

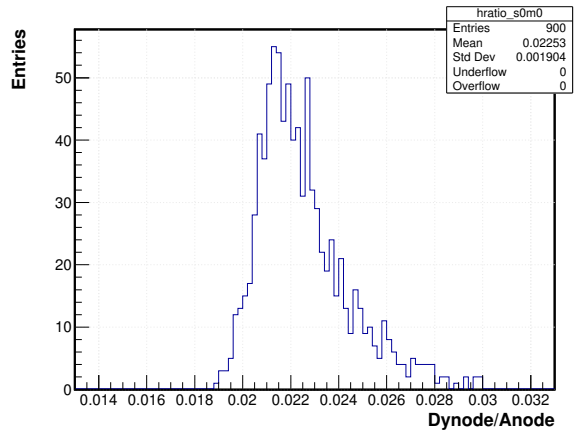

(b)

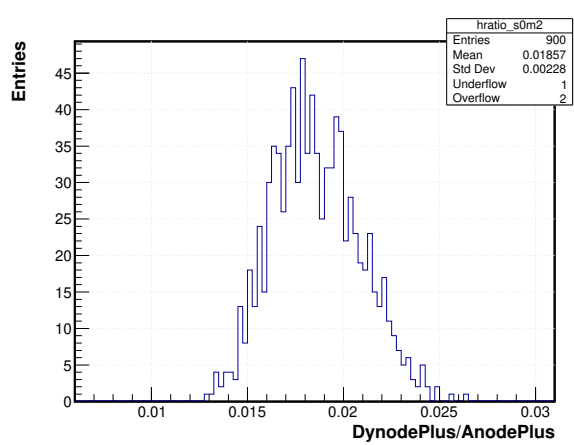

(d)

Figure 5: (a) Anode signals versus dynode signals of one typical 8-inch PMT. Inside the two green solid slashes is the fitting region. (b) Distribution of $D / A$ ratio of 900 8-inch PMTs in WCDA-1. (c) Anode signals versus dynode signals of one typical 1.5-inch PMT. (d) Distribution of $D / A$ ratio of 9001.5 -inch PMTs in WCDA-1.

WCDA-1 and others are in WCDA-2. For each pool, hits within the same loop are sorted by NPEs and tagged with rank from 0 to 1 . Then hits with same rank are paired. $\log _{10}(N P E s)$ of pairs of hits in the same loop are filled in a histogram. The ratio in each loop is obtained by linear fitting of the corresponding histogram as shown in Fig. 7. The final result is an average of the five closest of the nine ratios. Analyzing data from a single day in April 2021 yields a ratio of 11.78.

\section{Efficiency calibration}

The feasibility of using single particle peak to calibrate cell's detection efficiency for secondary particles has been revealed in previous studies [2]. In this article, the focus will be on the Constant Rate Scaling method. Under such assumption that integral energy flux detected by different cells is same over the same period of time, charge spectrum from single-channel data of two different cells should be exactly same if detection efficiency of the two cells is same. In fact, comparison of single channel charge spectrum shows that the detection efficiency of the cells in one pool is obviously different, mainly due to the difference of quantum efficiency and collection efficiency of 


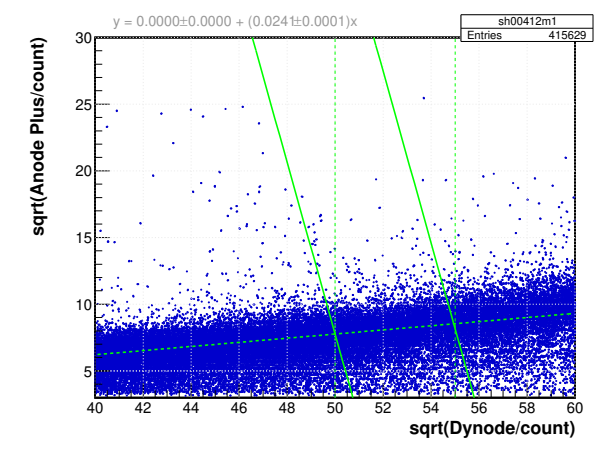

(a)

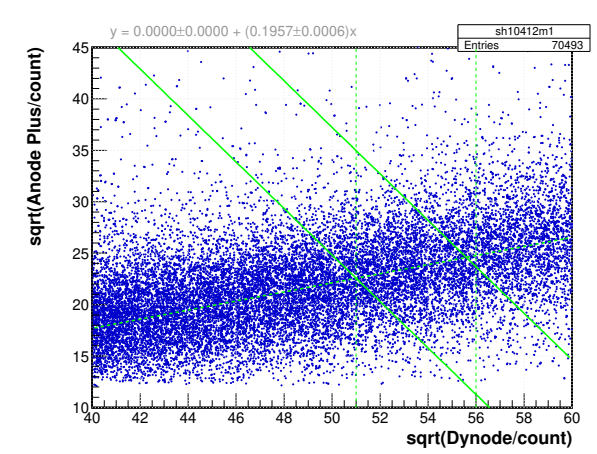

(c)

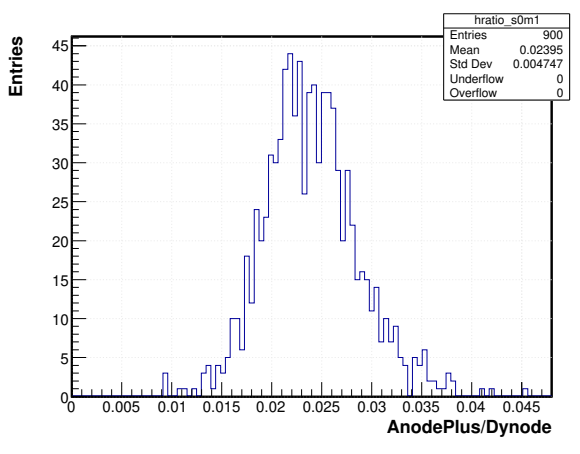

(b)

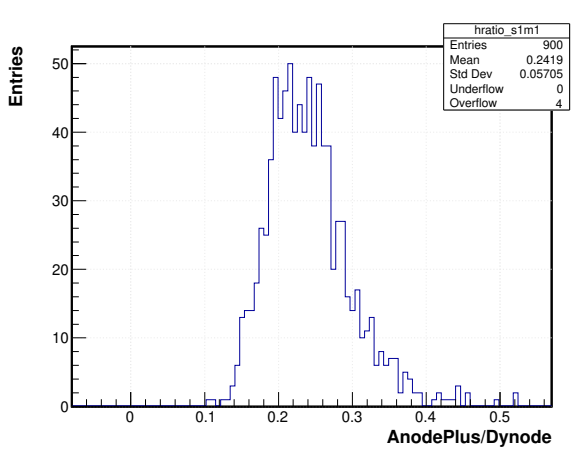

(d)

Figure 6: (a) Anode signals of 1.5-inch PMT versus dynode signals of 8-inch PMT. Inside the two green solid slashes is the fitting region. (b) Distribution of $A_{\text {plus }} / D$ ratio of 900 cells in WCDA-1. (c) Anode signals of 3-inch PMT versus dynode signals of 20-inch PMT. (d) Distribution of $A_{\text {plus }} / D$ ratio of 900 cells in WCDA-2.

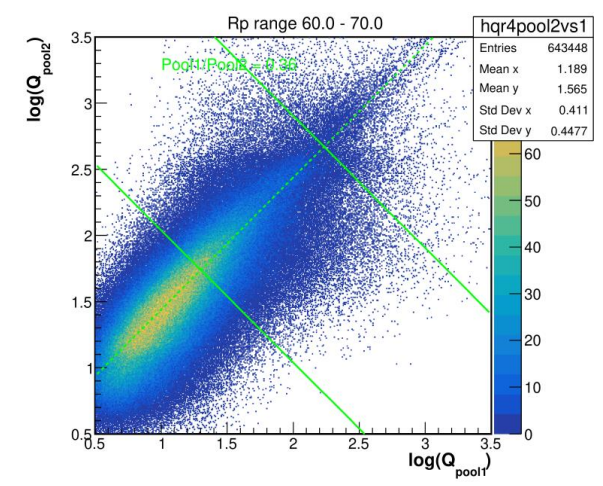

(a)

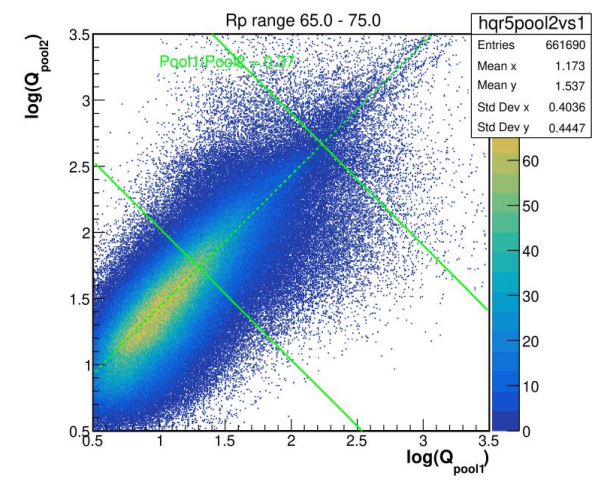

(b)

Figure 7: (a) $\log _{10}(N P E s)$ of hits in WCDA-1 versus $\log _{10}(N P E s)$ of hits in WCDA-2 in the loop $65 \mathrm{~m}$ to $75 \mathrm{~m}$ from the core. (b) $\log _{10}(N P E s)$ of hits in WCDA-1 versus $\log _{10}(N P E s)$ of hits in WCDA-2 in the adjacent loop. 
PMTs and the inhomogeneity of water quality. Therefore, we can quantitatively analyze the relative differences of detection efficiency based on the single channel charge spectrum.

In particular for the CRS method, the calculation of efficiency of "cell1" relative to the reference "cell0" is divided into three steps:

(1) an threshold in PEs (denoted as $t h_{i}$ ) is set for each cell, and $t h_{1}$ is equal to $t h_{0}$;

(2) adjust $t h_{1}$ until integral count rate above threshold of the two cells is equal;

(3) the efficiency of "cell1" relative to the reference "cell0" (denoted as $\eta$ ) is equal to $\frac{t h_{1}}{t h_{0}}$.

According to the change of water quality over time and single-channel hits rate, the cell 371 on 20190820 and cell 1458 on 20201110 are chose to be the reference of WCDA-1 and WCDA-2, respectively. The " $t h_{0} "$ are set to 15 PEs and 200 PEs. Preliminary results are shown in the Fig. 8. The results show that the relative difference between 900 cells is $0.0565 / 1.034=5.46 \%$ and $0.1189 / 1.006=11.82 \%$ for WCDA- 1 and WCDA- 2 .

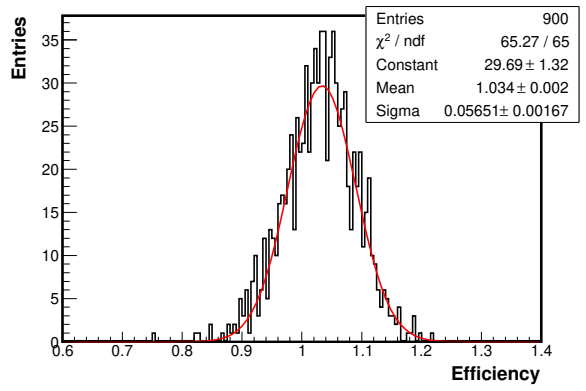

(a)

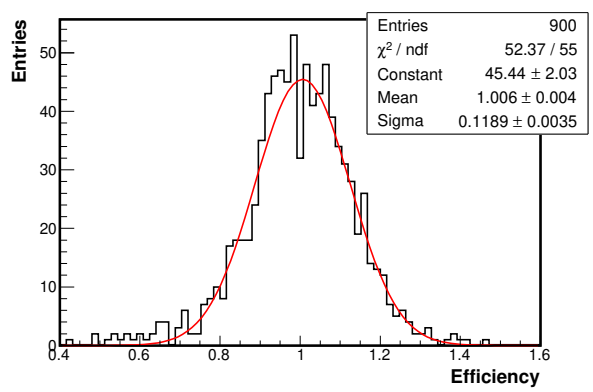

(c)

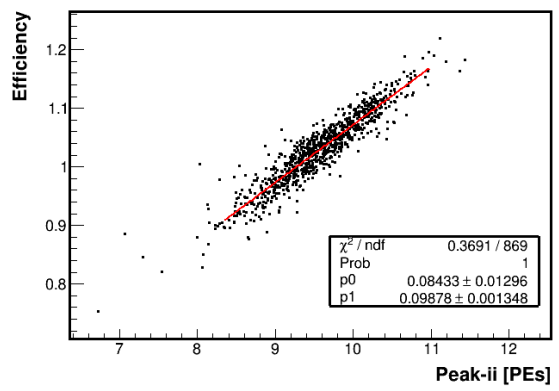

(b)

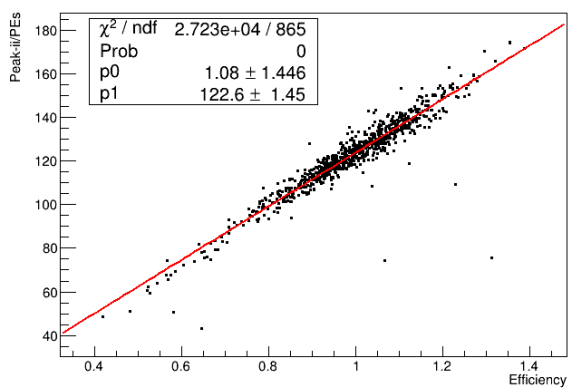

(d)

Figure 8: (a) Distribution of CRS efficiency of 900 cells in WCDA-1 on 20190820.The red line is the result of Gaussian fitting. (b) Efficiency versus single particle peak of WCDA-1. (c) Distribution of CRS efficiency of 900 cells in WCDA-2 on 20201110. (d) Efficiency versus single particle peak of WCDA-2.

\section{Summary}

We developed a relatively complete off-line charge calibration method, which can accurately connect signals of four types of PMTs, so as to achieve the purpose of combining three pools into one detector array. In addition, the comparison with the single particle peak shows that CRS method can effectively calibrate the efficiency. The application of results of CRS to subsequent data analysis is under study. 


\section{References}

[1] Z. Cao, A future project at tibet: the large high altitude air shower observatory (LHAASO), Chin. Phys. C, 34 (2)(2010): 249-252.

[2] H.C. Li, et al, A method to monitor and measure the water transparency in LHAASO-WCDA using cosmic muon signals, Chin. Phys. C, 41 (2)(2017): 026002.

[3] X. Li, X. Zhao, Z. Tang, C. Li, K. Jiang, J.B. Liu, Study of PMT performance in the high dynamic range at LHAASO, Nuclear Inst. and Methods in Physics Research, A 912 (2018) $382-385$.

[4] H.Y. Zhang, Y.H. Yu et al., Photomultiplier tube performance of the WCDA++ in the LHAASO experiment, Nuclear Inst. and Methods in Physics Research, A 958 (2020) 162079. 